\title{
Role of a Teacher in developing socio-emotional skills of Preschoolers
}

\author{
Deepika Rajawat ${ }^{1}$ \\ ${ }^{l}$ (Department of Education(CASE),The Maharaja Sayajirao University of Baroda, India)
}

\begin{abstract}
What do we want our children to be when they grow up- a literate, responsible, non violent, good character, caring adult ?Each of these elements of challenge can be enhanced by thoughtful, sustained and systematic attention to the social and emotional skills of children at a stage as early as preschool. Because this is the stage when almost 85\% of the development of brain is complete in this period of 0-6 years and whatever experiences that are gained in this span, shapes the future of the child. Children are like clay and can be molded into any desired shape, so let this desire be not only, of a child who is good in academics but also a good human being or a holistic child. Social emotional skills like (self awareness, self management, social awareness, relationship skills and responsible decision making) helps the children in better adjustments, better transition and better performance not only in schools but throughout their life. Social-emotional learning is sometimes called 'the missing piece', because it represents a part of education that links academic knowledge with a specific set of skills important for success in schools, families, communities, workplaces and life in general. It is quite unfortunate when children grow up with knowledge but without social-emotional skills and a strong moral compass. Hence, a combination of academic and social emotional learning is the true standard for effective education in the world today and for the foreseeable future. But what kind of competence is required on the part of teachers (for School readiness, Adjustments, Social relationship, Individual development, Self and social awareness, responsible decision making etc.) to bring this kind of learning among preschool children that will help them to grow into a good or successful human being needs to be pondered over.
\end{abstract}

Keywords: Social Emotional Skills, Preschool Education, Preschoolers, Early principles of learning

\section{Introduction}

J. B. Watson $(1925)^{[1]}$ said "Give me two infants at the impressionable age. I can make them an Einstein or a Hitler or a Marconi by providing suitable environment". May be he was too optimistic but no one can deny the power of environment, the experiences, the opportunities that we give to a child helps in shaping his future. India is home to more than one billion people, of, which $42 \%$ are children, defined as persons less than 18 years of age. Nineteen percent of world's children live in India. There are about 43 crore children in the age group of 0-18 years, of which about 16 crore are represented by the young child under 6 years of age. There is consensus that the early years are the most valuable and vulnerable period when there are the greatest risks to survival, healthy growth, development and susceptibility to a vicious cycle of under nutrition and disease/ infections. These early years are also crucial for cumulative lifelong learning \& human development through physical, cognitive, emotional, social and linguistic development (Report of the working group on child rights for the $12^{\text {th }}$ five year plan (2012-2017) $)^{[2]}$. Now if this huge chunk of population (16 crore) is taken care nicely they can be moulded easily to become the future faces of the nation. For this preschool is the initiating point.

\subsection{Preschool Education}

Preschool education is a term used in common parlance to denote a variety of early childhood care and education (ECCE) programmes that provide opportunities for the quality care and education of the children between the age of two and six years. It is viewed in a very broad perspective and encompasses all aspects related to children's development and education. In India, the term is used interchangeably with early childhood education (ECE) and Pre-primary education and covers within its ambit a variety of Nursery and Kindergarten school as well as government sponsored programmes like Balwadis and Anganwadis.(Sharma, 2006) ${ }^{[3]}$

The teacher during preschool has to be sensitive to individual difference and pace of development of children when helping children grow and learn. Thus it is important to know the Characteristics of the preschoolers which are as follows:

\subsection{Characteristics of the Preschoolers}

3 yr old: Physically, the three year old is independent in most routine activities like brushing teeth, taking bath, toilet management and eating. The child's large muscles have increased in strength and coordination, but lack agility.The ability to co-ordinate small muscles as well as eye and hand coordination increases. 
Three year old is a talkative child as long abilities increase during this period. The child's conversation is a collective monologue and the child may not express hishher thoughts in continuity with ongoing conversation but will speak his her thoughts irrespective of the topic of conversation. The child is verbal enough to express basic needs and ideas and is extremely fond of listening to stories, songs and music. However the attention span is limited and children in this age group prefer to engage in a variety of activities for a brief period of time. The child enjoys participation in activities and assisting in small chores, constantly seeking adult attention approval, guidance and interaction. The child likes to play with peers but may not interact actively and generally indulges in second plan.

$4 \mathrm{yr}$ old: At four, the child is more capable of managing himself and independently satisfying his physical needs. The child needs little assistance in eating, bowel control, moving, climbing etc. The child uses proper long to interact with others, ask questions and make known feelings, needs, and ideas. In fact the four year old can be very attention seeking may show off given the opportunities and may be aggressive and loud in making his presence felt. The child has developed a definite personality and can make his own decisions in daily chores. The child's gross motor movement is well co-ordinated. The child has better control over fiber muscle movement and can hold chalks/crayons, blocks, pencils etc to draw the paint and use scissors for cutting. The four year old child enjoys Peer Company. The child is rapidly learning to play cooperatively with playmates of his age. The child uses realistic props, using tools, utensils and enjoys dramatic play. This signifies development of larger attention span and broader interests.

5 yr old: Generally five year old child has good physical control. He can throw, jump, catch, skip, and use scissors and crayons with precision. The child is ready to begin learning the $3 \mathrm{R}$ 's since he can also remember and understand the sequence of things, numbers and alphabets. The child command over language is comprehensive and is used to express feelings, ideas and complying with requests. Attention span has increased considerably and so has eye hand as well as fine muscle co-ordination. Thus, the five year old is independent, dependable and self assured. He likes to get constant approval and assurance from others. The child enjoys playing with same sex and age children. Play is sustained, cooperative and complex and may last from one day to another. (Position Paper ECCE, 2006) $)^{[4]}$

Thus looking at the above characteristics of the preschoolers, it is very important to understand the development of children. Thus the teachers recruited in the preschools should be qualified to understand the psychology and development of the children. Only females are considered for preschools as the children feel comfortable with a motherly figure in this age. In regard with educational qualification, the teachers should have either of these, diploma in ECCE/CD, B.Sc. in home science or Masters in any subject. The preschools aim to bring an all round development among children. This kind of development is possible when each and every aspect of a child is considered i.e. socially, mentally, emotionally, physically etc. In today's world, a combination of academic and social emotional learning is the true standard for effective education and for the foreseeable future, where Teachers play the most important role. Thus it is necessary to know about the role they play in the life of Preschoolers for their socio-emotional learning and development.

\subsection{Social Emotional Learning (SEL)}

Social Emotional Learning (SEL) is defined as the process of acquiring a set of social and emotional skills- self awareness, self management, social awareness, relationship skills and responsible decision making within the context of a safe, supportive environment that encourages social, emotional and cognitive development and provide opportunities for practicing social-emotional skills (Cherness, Extein, Goleman, \& Weissberg, $2006)^{[7]}$. SEL is a process for learning life skills, including how to deal with oneself, others and relationships, and work in an effective manner. In dealing with oneself, SEL helps in recognizing our emotions and learning how to manage those feelings. In dealing with others, SEL helps with developing sympathy and empathy for others, and maintaining positive relationships. SEL also focuses on dealing with a variety of situations in a constructive and ethical manner. These competencies provide the foundation for maintaining high-quality social relationships and for responding to the challenges of life. The teachers other than parents are the only people who can enhance the SEL among preschoolers, but they need certain competencies to achieve so.

\section{Role of Teachers}

Teachers can accomplish this in the classroom through effective and direct classroom instructions, student engagement in positive activities, and involving parents, students and the community in planning, evaluating and implementing the program into the classroom. The first word that a child learns is of a mother or father, so both of them play a very crucial role in providing early childhood care. Various findings have revealed that family income, young maternal age at child's birth, mother's educational attainment, number of siblings in a household, parents involvement, family conflicts and family resources and their attitude towards education all 
these influences the child development and his/her education. At school safety and security in terms of health, love, care, attachment and infrastructure, should be taken care off and teachers are the second parents to do so or achieve so. If the basic need of child that is safety is not taken care of, it will create fear in the child, thus hindering his/her growth, development and learning. Faulty methods of teaching, like focusing only on academics(3Rs), disregard of individual differences, lack of appropriate use of play materials, lack of activities leading to holistic development and Lack of co-curricular activities can also affect his her learning and development. Emotionally unbalanced teacher can cause havoc, as the teacher plays a role model for children and they imitates him or her. So what kind of skills a preschool teacher should have during this crucial stage of development of a child during his/her transition from home to school and how these skills can help the children in their social and emotional learning will be focused here by the researcher.

\author{
2.1 Competencies Required In Preschool Teachers for Socio-Emotional Development of Preschoolers \\ 1. Emotionally balanced \\ 2. Knows vernacular medium \\ 3. Sound Knowledge of Psychology and Child development \\ 4. Knowledge of all the Principles of Early Learning and its effective implementation \\ 5. Understands the individual needs of the children \\ 6. Be able to deal with all sorts of emotions (anger, sadness, happy) and help them in self and social awareness \\ 7. Develops relationship with and among children \\ 8. Teaching children to problem solve and decision making \\ 9. Make rules in the form of observable behaviour \\ 10. Find out ways to motivate children \\ 11. Use different modalities for learning \\ 12. Integrate play with learning \\ 13. Involve parents in learning \\ 14. Circle play \& circle song
}

\title{
1. Emotionally balanced:
}

A teacher should know how to handle her emotions first, and then only she'll be able to deal with young kids. In order to reach to their level and understand their psychology she should be stress free and happy. She should be able to shut herself off from her environmental disturbances so that she could give the young and tender children the love, care and affection they deserve. She should be able to develop trust and belongingness in them (belongingness to school and to her) and this is possible only when they feel like home at schools. The teacher in the preschools is a role model to children. Thus a teacher should be very careful about the way she acts with the kids and should actually be a motherly figure to them.

\section{Knows vernacular medium:}

The language at home is the birth language of kid. The transition from this L1 language to medium of instructions at school (L2) is not easy for the kids. So the teacher needs to use L1 along with L2 and then slowly bring them to L2. This transition might take a long time but in this way it will become a smooth one for them.

\section{Sound Knowledge of Psychology and Child development:}

The preschool teacher should be all updated with child developmental theories and their applications. What Jean Piaget, J.B.Watson, Lev Vygotsky, Maria Montessori, Froebel, Eric Erikson, Albert Bandura, John Bowlby, Howard Gardener, Lucy Sprague Mitchell etc have said and given should be followed.As well as about the characteristics of children at the early childhood stage. They should try and understand the psychology of the child, his/her needs, family background, culture from which s/he comes, learning style, his/her individual needs and how can a teacher give them a homely environment, lots of love, care and belongingness.

\section{Knowledge of all the Principles of Early Learning and its effective implementation:}

- Learning begins from birth: From infancy children are mentally and physically active. They learn through all their senses and stimulations.

- Children construct knowledge: They construct their own knowledge while they actively engage in their environment and through repeated interactions with people and materials. This simply means that children touch, taste, shake things etc. to find out what happens and learn about things in their environment.

- Children's curiosity and desire to learn: Children are curious and eager to learn. They have an inherent need to make sense of their experiences and learn about the world around them.

- Children learn through play: Play is central to the child's well-being and development Children's spontaneous play provides opportunities for exploration, experimentation, manipulation and problem 
solving that are essential for constructing knowledge. Play contributes to the development of representational thought.

- Child development and learning are characterized by individual variation: No two children are same.

- Children's learning reflects a recurring spiral that begins in awareness, and moves to exploration, to inquiry, and finally, to application.

- Children need to experience success more than failure to form a positive self-concept: The experiences should be planned in accordance with the maturational level of the children, such that they are challenging yet achievable, so as to promote self-confidence.

- Children develop holistically and benefit from integrated experiences and education: All the domains of development i.e. physical, motor, cognitive, language, socio-personal, emotional and creative and aesthetic appreciation are interrelated and take place simultaneously. (draft 2012) ${ }^{[5]}$

\section{Understands the individual needs of the children:}

Each child is gifted with a different ability and different need. It is up to a teacher to identify and bloom it. E.g. some child can be a quiet child, some impulsive, some can be a lonely child, some with high IQ and some with low. But being a quiet child s/he might be very creative. The teacher should target the particular behaviours she wants to strengthen in each individual child. She may plan to praise the quiet, withdrawn student every time s/he ventures an answer or participates. In the case of preschool children it is developmentally normal for 3-5 year olds to be impulsive and to have difficulty waiting. With this age children the goal of a teacher may be simply to encourage their participation and attention. But gradually when the teacher responds primarily to quiet hands up and reinforce this behaviour (and ignore blurt outs) one can help these children learn that this is expected classroom behaviour. So every child might have different needs and talent which needs to be identified by the teacher and encouraged.

\section{Be able to deal with all sorts of emotions (anger, sadness, happy) and help them in self and social awareness:}

The teacher should be sensitive enough to recognize the everyday emotions of a child. Suppose a child is sad then through her love and affection or by talking or involving in some fun activities she should be able to bring her/him back. Preschoolers are very young and they tend to forget things very fast. In this similar way if the child is angry, one can make them follow turtle technique to cool off. Happy child can be asked about her happiness and can be shown to others so that they learn that it is good being happy. Various feeling charts can be used to teach them different emotions and how to deal with them for eg anger as mentioned above using turtle technique that is putting head in the shell, then waiting for few seconds and take your head out when you have cooled off. Playing games of doctor and 'asking them how they are feeling today?' or ask them to identify their emotion on feeling charts. Making them watch in the mirror and ask them to make faces or say what they are feeling? but it should be first shown by the teacher in the form of observable behaviour. Making them feel about the various emotions through role play etc. This will help them in developing empathy for others. Children should be taught to help the elderly person in their homes and community, help their parents in cutting vegetables or fruits, take out things from refrigerator, arranging dining table for dinner, carrying small grocery bags etc. They should be asked to pick up the waste from their school grounds or to keep their classrooms clean. This will make them feel responsible.

\section{Develops relationship with and among children:}

First and foremost the teacher should develop relationship individually with every child. the attachment building with children gives them a sense of safety, security and trust which is very important for their development. Then to develop social relationship and for social learning, group tasks should be given to them so that they learn helping and believing each other, sharing, cooperation etc. the groups can be formed in various ways like on the basis of the birthdays within same months or similar first alphabets of their names or through their favorite animal names etc. in this way every time they come in contact with new person and make new friends. Initiating circle games increase social awareness and peer praise. Giving them everyday some time for "free play" also enhances their social skills because in the "free play" they form groups on their own, they get the right to choose with who so ever they want to play with. During free play, teacher should give them lots of options i.e. blocks, doll house, kitchen sets, learning material etc and then leave them to play with any of the materials they want to play with. It is quite interesting to watch them play and see the formation and reformation of groups

\section{Teaching children to problem solve and decision making:}

The teacher is already teaching the students more appropriate problem solving tactics without realizing it. The other ways through which the teacher can do it is using puppets to role play problem situations. Teacher can develop a script taking any one problem suppose 'Heeral getting teased' so there can be conversations between 'Heeral' and 'teacher' in the script and that can be presented through puppets in a role play. In this way 
various scripts can be developed for various problems like "being angry is not good", 'picking up fight is bad', 'not sharing', 'it's mine', 'she won't let me play with her', 'she hit me' etc. the scripts should be such that it makes the children think. The students can also be explained about the conflict situations which they face every day with their friends in the classroom. The teacher can also prepare the students for these scripts and let them only present it to their peers. It is more effective.

\section{Make rules in the form of observable behaviour:}

Classroom rules and expectations should be clearly spelled out, posted in the classroom, and reinforced when students follow them. There should be no more than five to seven rules that are stated in positive terms. For e.g., a rule such as 'stay in your seat' is clear, whereas 'no fooling around' is vague and focuses on something negative. The rules should be stated in terms of observable behaviours, which are behaviours you can see. For e.g. 'Keep your hands to yourself' is preferable to 'show respect' or 'be nice' because a child will have a clear mental image of what specific behaviour is expected. Similarly 'complete your homework and put it on my table next morning' is preferable then 'be responsible about your homework'. Rules such as 'be responsible' or 'be a good citizen' are ineffective because they are ambiguous and it is unclear what behaviours are being asked for?

\section{Find out ways to motivate children:}

Children should be encouraged and praised through various incentives like giving stars on their palms, putting feather in their cap, giving special awards, using daily behavior charts but for it the teacher should have clearly identified the positive behaviors that she will put on the chart. It is important that these behaviors be explained clearly. It needs to be modeled and practiced or rehearsed. For eg. in a situation like, "when a student was tying the lace of another student....the teacher made all the children to stop doing what they were doing and showed them what those two students were doing. then she took their names, explained what a wonderful way it was to help a friend and asked the class to clap for them and pasted shimmering stars on the back of their palms." so in this way children got motivated to repeat the behaviour while other students started offering their help in tying the shoe laces of that kid.

\section{Use different modalities for learning:}

The teacher should be enthusiastic and creative and should use different modalities every day for learning. E.g. role-play, dramatics, poems, dance, art and crafts etc. thus the interest of children is maintained in this way in the academics. Preschoolers have a very short attention span so new constructive and creative things are required on the part of teacher to keep the children engrossed and help them to learn. Various play materials are required like beads of different colours and size, clay and clay moulds of different colour for children to make different shapes with them, building blocks, different geometric shapes, coloured paper, scissors, tapes etc; to make learning more fun.

\section{Integrate play with learning:}

There are many preschools which use play way method of learning. But are they really able to integrate the play with learning? The outdoor games are very important and the teacher needs to direct them for better learning but this doesn't mean giving them strings of commands and corrections that make the experience unpleasant for children. First of all the teacher in the free play times with students is to follow their lead, ideas and imagination. Imitate their actions and do what they ask you to do. For e.g. if the children wants to play 'the red letter A' where the child has to take total steps according to the number of times the alphabet is there in its name, the teacher can also play with them. The other e.g. is 'Simon says' in a circle and then the child has to do what Simon says etc. if the teacher is not playing she can be an appreciative audience.

\section{Involve parents in learning:}

The teacher should be aware of the personal conditions or home environment of each child. Then only she will be able to do better with children. The teachers should inform the parents about the skills being taught to their children so that they can take care of it at their respective homes. They should be informed about their daily schedules and routines so the parental involvement is there in the learning of children. The parents can even be called at schools to get involved in certain role plays and activities. The teacher can make use of the professions of the parents and ask them to take a class when the topic "community helpers" goes on. For example a student whose parent is a doctor can come and talk to them about their profession by enacting or through role play. similarly a banker, a lawyer, a teacher etc can contribute to their children's class. This makes children feel more comfortable and enthusiastic. The teacher can even pay home visit if she feels she should. 


\section{Circle play \& circle song:}

The teacher should involve the students in activities through circle play-way learning and circle songs. Like passing the box in a circle and when the music or 'daphli' stops the student holding the box talks. This develops their self concept, confidence, enhances social and emotional development etc. With this circle play way learning they become so attentive and alert and even keep holding the box instead of passing so that when the music stops, they have the box in their hand or they get the chance to speak. It is like a big win for them.

\section{Conclusion}

Thus in the end we should not forget that the preschool teacher has a great responsibility of moulding and shaping the children's future as it is the most influential stage. The young child's development proceeds rapidly in the early years: the basis of language is laid at this time; attitudes and social relationships are shaped; and the mind is at its most receptive (Rao, 2002). Knowing the importance of early years and their social and emotional development along with academics, the preschool education and the role of teachers becomes very important and unavoidable. The Preschool teachers when dealing with preschoolers have to be Emotionally balanced, should Know vernacular medium, should have the Sound Knowledge of Psychology, theories of learning and Child development, Knowledge of all the Principles of Early Learning and its effective implementation, Understands the individual needs of the children, their cultural context during teaching and learning process, help the children in understanding their emotions (anger, sadness, happy, jealousy, empathy etc) and in self and social awareness, Develops relationship with and among children, teach them how to deal with conflicts and resolve them, help them take decisions, Make rules in the form of observable behaviour, act as a mother and a role model, motivate them, Use different modalities for learning like Circle play \& circle songs, Integrate art and play with learning, Involve parents in learning etc

The thoughts and ideas reaped in this age remains throughout their life and as it is said "We sow what we reap". Thus if the teacher sows the best, the best will come out in future in the form of a literate, responsible, non violent, good character, caring adult.

[1]. Watson, J. B. (1925). Behaviourism. New York: Norton.

[2]. Government of India. (2012-2017). Report of the working group on child rights for the $12^{\text {th }}$ five year plan (2012-2017), New Delhi: Ministry of Women and Child Development.

[3]. Sharma, R. (2006). Preschooling in India and its impact on education. Delhi: Vista International Publishing House.

[4]. NCERT. (2006). Position Paper: National Focus Group on Early Childhood Education. New Delhi: National Council of Educational Research \& Training.

[5]. Government of India. (2012). Early Childhood Education Curriculum Framework Draft. New Delhi: Ministry of Women and Child Development.

[6]. Sharma, B.M., \& Sharma, A.S. (2009). Encyclopedia of Education in $21^{\text {st }}$ Century: Comparative Education, Vol. 4. New Delhi: Commonwealth Publishers.

[7]. Cherness, C., Extein, M., Goleman, D., \& Weissberg,R.P.(2006). Emotional Intelligence: What does the research really indicate? Educational Psychologist, 41(4), 239-245. 